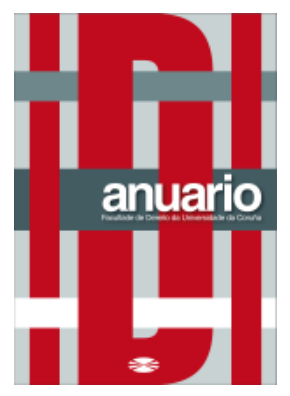

Anuario da Facultade de Dereito da Universidade da

Coruña Vol. 24 (2020), pp. 83-97

ISSNe: 2530-6324 || ISSN: 1138-039X

DOI: https://doi.org/10.17979/afdudc.2020.24.0.7462

\title{
EL DERECHO AMBIENTAL Y EL CONSTITUCIONALISMO EUROPEO
}

\section{ENVIRONMENTAL LAW AND THE EUROPEAN CONSTITUTIONALISM}

\author{
ANTONIO MANIATIS \\ Exdocente de la Universidad de Castilla-La Mancha
}

Recibido: 01/08/2020

Aceptado: 25/09/2020

\begin{abstract}
Resumen: El derecho ambiental constituye una rama genérica de derecho mientras desde hace 2015 la disciplina del derecho del cambio climático ha aquistado autonomía frente a esta rama. Francia es el único país europeo que ha consagrado explícitamente el principio constitucional de precaución, en la Carta del Medio Ambiente en 2005 y el primer país que prohibió el uso del fracking, invocando la precaución, mientras los jueces constitucionales europeos han hecho un uso raro de este principio. De manera similar, un nuevo principio, relacionado con la endogeneidad ambiental de actividades como es el turismo, podría consagrarse. El desafío para el futuro del constitucionalismo europeo y global sería prohibir en las Constituciones el uso del fracking y consagrar los derechos fundamentales universales al Sol, sobre todo a la mitigación del cambio climático.
\end{abstract}

Palabras clave: Constitucionalismo, Derecho ambiental, Derechos al Sol, Endogeneidad ambiental del turismo, Fracking / craqueo hidráulico, Precaución.

Abstract: Environmental law constitutes a generic branch of law whilst since 2015 the discipline of climate change law has acquired autonomy against this field. France is the unique European country to have explicitly consecrated the constitutional principle of precaution, in the Charter for the Environment in 2005, and the first country to prohibit the use of fracking, by invoking precaution, whilst European constitutional judges have made a rare use of this principle. In a similar way, a new principle, related to the environmental endogeneity of activities such as tourism could be enshrined. The challenge for the future of European and global constitutionalism would be to prohibit in Constitutions the use of 
fracking and to consecrate the universal fundamental rights to the Sun, mainly the right to Containing Climate Change (CCC).

Keywords: Constitutionalism, Environmental law, Solar rights, Environmental endogeneity of tourism, Fracking / hydraulic cracking, Precaution.

Sumario: I. INTRODUCCIÓN AL DERECHO AMBIENTAL. II. EL DERECHO AMBIENTAL DESDE EL PUNTO DE VISTA DEL DERECHO PÚBLICO. III. EL DERECHO DEL CAMBIO CLIMÁTICO. IV. EL PRINCIPIO DE PRECAUCIÓN Y LA JUSTICIA CONSTITUCIONAL EUROPEA V. LA CUESTIÓN DE PROHIBICIÓN DEL FRACKING Y LA JUSTICIA CONSTITUCIONAL EUROPEA VI. LA ENDOGENEIDAD AMBIENTAL DEL TURISMO. VII. EL DERECHO AMBIENTAL EN LA VANGUARDIA DEL DERECHO CONSTITUCIONAL. BIBLIOGRAFÍA

\section{INTRODUCCIÓN AL DERECHO AMBIENTAL}

El medio ambiente constituye una materia multidisciplinaria, en conexión estricta con la cultura, incluyendo el medio ambiente antropogénico. El derecho ambiental es una rama autónoma, la cual ha adquirido una estrecha relación con la normativa constitucional. El constitucionalismo ambiental se enmarca en el abordaje de la cuestión de cómo las disposiciones constitucionales impactan la calidad ambiental y los derechos ambientales de los ciudadanos ${ }^{1}$. Más precisamente, tiene que ver con las garantías de cada persona relacionadas con el medio ambiente y con el papel del Estado en esta materia. En otras palabras, la normativa constitucional no se limita a la misión de las autoridades públicas, sino que también afecta a los individuos.

Como ya sugerido, la temática del derecho ecológico es muy amplia, como es el caso de las disposiciones constitucionales sobre el medio ambiental. El presente estudio analiza de manera indicativa algunos aspectos de este derecho y del constitucionalismo, particularmente del constitucionalismo europeo. Se refiere a varios ordenamientos nacionales de países europeos, dotados de una normativa y jurisprudencia importante en esta materia, y también al derecho de la Unión europea y al derecho internacional ${ }^{2}$.

En primer lugar, examina el derecho ambiental desde el punto de vista del derecho público. La aproximación a esta rama se realiza en el campo del derecho constitucional y también del derecho administrativo.

${ }^{1}$ HUDSON, B., “Structural Environmental Constitutionalism”, Journal Articles. 2015, 170, p. 201, https://digitalcommons.law.lsu.edu/faculty_scholarship/170.

2 MANIATIS, A., "PPP and the constitutional right to the environment", Constitutionalism.gr, 20.11.2015, $\quad$ https://www.constitutionalism.gr/ppp-and-the-constitutional-right-to-theenvironment/?hilite=\%27Antonios $\% 27 \% 2 \mathrm{C} \% 27$ Maniatis $\% 27$. 
La temática del derecho ecológico se amplía a través de una normativa más reciente. Se trata del derecho relacionado con la mitigación del fenómeno del cambio climático.

Después de esta parte general, el análisis se centra en el principio de precaución, el cual constituye ello mismo un elemento novedoso y avanzado en el marco del derecho ambiental. El acercamiento a esta conquista del constitucionalismo europeo se realiza en combinación con la jurisprudencia de la jurisdicción superior y constitucional de varios países.

El estudio de la herramienta precautoria se proyecta en la cuestión de la prohibición del craqueo hidráulico (o fracking). Se trata de una amplificación de la aproximación anterior siempre en conexión con la jurisprudencia constitucional europea.

Finalmente, la problemática del principio precautorio, como completada con el análisis jurídico del fracking, podría conducir a consideraciones alternativas sobre la metodología del desarrollo económico, sea a nivel nacional o regional. Existe el dilema de un desarrollo basado en la intervención técnica con efectos inciertos o claramente negativos sobre el medio ambiente o amigable con él, mucho más “internalizado”. Una rama la cual está denominada “derecho ecológico” merecería una orientación ecológica avanzada, ejemplificada por el principio de endogeneidad ambiental del turismo.

\section{EL DERECHO AMBIENTAL DESDE EL PUNTO DE VISTA DEL DERECHO PÚBLICO}

En Francia y en Grecia los primeros conjuntos de reglas especiales (y no más de disposiciones dispersas) en materia de las relaciones de la gente con los bienes ambientales emergen en $1976^{3}$. Se trata de la adopción de leyes relativas a la protección de la naturaleza, del urbanismo y etc. en el ordenamiento francés mientras en Estrasburgo tuvo lugar la primera conferencia de la Sociedad Francesa del Derecho del Medio Ambiente. Paralelamente, el parlamento griego votó la ley núm. 360/1976 “de planificación espacial y medio ambiente".

Es notable que la primera invocación de una rama de derecho ambiental es posterior a la creación de instituciones especialmente dedicadas a la protección del medio ambiente y que este derecho corresponde a las funciones de un nuevo ministerio, llamado “Ministerio de la Protección de la Naturaleza”, el cual fue creado en Francia en 1971. De modo similar, Grecia adquirió el Ministerio de Planificación Espacial, Vivienda y Medio Ambiente con la ley núm. 1032/1980. Una vez creada una nueva institución administrativa que produce derecho y tiene como propósito la protección ambiental, una nueva rama de derecho se debía reconocer. Así la opinión del doctrinario francés M. Hauriou sobre la institución administrativa como organización que está sujeta a una idea, fue confirmada de

${ }^{3}$ SIOUTI, G., La consagración constitucional de la protección del medio ambiente, Ediciones Ant. N. Sákkoula Atenas - Komotiní 1985, p. 14 (en griego). 
nuevo ${ }^{4}$. En otras palabras, se constata un desarrollo paralelo de las dos subramas del derecho público, es decir del derecho constitucional y del derecho administrativo, en materia de la creación y del desarrollo inicial del sistema jurídico y administrativo ambiental. En términos constitucionales, el Estado adquirió una “obligación especial”, consistente en la protección de los bienes ambientales. Este deber se proyectó específicamente en el campo de la misión de la Administración Pública; en términos del derecho administrativo, el medio ambiente correspondió a un nuevo "servicio público”.

Por lo general, la noción del servicio público, como señalado por el Tribunal Constitucional español, no constituye una noción unívoca y sí un concepto muy debatido por la doctrina - con detractores y valedores - o sujeto a distintas elaboraciones y utilizado en diversos momentos históricos con finalidades también distintas ${ }^{5}$. Hay un enfoque conceptual, que consiste en el criterio objetivo (actividad) y en el criterio subjetivo (organismo público). La idea básica que estableció la sentencia Romieu de 6 de febrero de 1903, coincidente con la jurisprudencia del Consejo de Estado francés y la doctrina más autorizada, es que el servicio público existe con un régimen jurídico especial dentro del derecho público, absolutamente alejado del derecho privado.

La rama genérica del derecho ambiental, como aquella - en cierto modo “especial”- del derecho de la cultura, podría considerarse como nueva rama de la parte especial del derecho administrativo. Dado que tiene también aspectos constitucionales, como ya señalado, este fenómeno es relacionado con la distinción entre el derecho constitucional y el derecho administrativo. En la concepción unitaria de estas dos disciplinas reside la exigencia de que la actividad de los poderes públicos no estuviera desvinculada de los principios fundadores del ordenamiento ${ }^{6}$.

Además, se han usado tres adjetivos para comprender el dinamismo del derecho ecológico ${ }^{7}$. Se trata simultáneamente de un derecho intrínsecamente complejo, compuesto, por lo que se refiere a varias disciplinas clásicas del derecho, y expansivo, dado que tiende a tratar ciertas ramas nuevas y que está vivificado por el progreso de disciplinas jurídicas especializadas (derecho de energía, derecho de productos, derecho incipiente de la economía circular). En todo caso, sigue siendo en interpelación permanente con los avances del conocimiento científico (las nanotecnologías, las biotecnologías, la biodiversidad para los equilibrios biológicos).

\section{EL DERECHO DEL CAMBIO CLIMÁTICO}

4 DE LANVERSIN, J., "Contribution du juge au développement du droit de l'environnement”, en Mélanges M. Waline - Le juge et le droit public, T. II 1974, pp. 521-522.

${ }^{5}$ FEIJÓO REY, M. J., y PIMENTEL SILES, M. (Editores), El interés general y la excelencia en los servicios públicos. La mejor gestión de los servicios municipales en favor de los ciudadanos y no de las ideologías, Almuzara, 2017, pp. 73, 74.

${ }^{6}$ ROMANO, A., “Los principios constitucionales de las Administraciones Públicas”, ReDCE núm. 23, 2015 (trad. PÉREZ MIRAS, A.).

7 HUGLO, CH., “La QPC: quelle utilisation en droit de l'environnement?”, Nouveaux Cahiers du Conseil Constitutionnel, n. 43 (le Conseil constitutionnel et l'environnement) avril 2014, https://www.conseilconstitutionnel.fr/nouveaux-cahiers-du-conseil-constitutionnel/la-qpc-quelle-utilisation-en-droit-de-lenvironnement. 
La palabra “clima” procede del latín “clima” y éste del griego “klima”, que significa inclinación del Sol y por extensión latitud, clima. El cambio climático consiste en la alteración del clima mediante el calentamiento, ejemplificada por el cambio muy frecuente del tiempo en una región. Si este fenómeno tiene algunos aspectos positivos, particularmente para algunas partes del planeta, incluye aspectos sobre todo negativos. Comenzó a preocupar a la comunidad internacional en el fin de la década de 1980. La conferencia mundial sobre el cambio de la atmósfera tuvo lugar en 1988, con la iniciativa de la Organización Mundial de Meteorología.

El primer tratado internacional sobre el cambio climático se concluyó en 1992 y entró en vigor el 21 de marzo de 1994. Se trata de la Convención Marco de las Naciones Unidas sobre el Cambio Climático, ratificada entre otros por la Unión europea. Ha resultado un derecho nuevo en la materia, de la cuarta generación en la historia de consagración de los derechos fundamentales ${ }^{8}$. Esta generación consiste en varios derechos en vía de reconocimiento, como los relativos a las manipulaciones genéticas y la bioética, a las nuevas tecnologías de comunicación y al mundo de los animales ${ }^{9}$.

Después de este texto, sigue una serie de conferencias de las partes contratantes, conocidas como “Conference of Parties (COP)". En 1997, de la “COP 3” resultó el Protocolo de Kioto, el cual completa el contenido de la Convención, fijando algunos objetivos vinculantes, como reducir las emisiones de seis gases de efecto invernadero que causan el calentamiento global. En el protocolo se acordó una reducción de al menos un 5\% de las emisiones de estos gases por el período 2008-2012 frente a las emisiones de 1990. En 2012, durante la “COP18” en Doha, fueron adoptadas las modificaciones del Protocolo, en materia del segundo período de aplicación, 2012-2020.

La doctrina señaló en 2015, antes de la “COP21”, que la disciplina del derecho del cambio climático estaba aquistando autonomía en el ámbito del derecho ambiental ${ }^{10}$. Al menos tres eran las revistas jurídicas dedicadas en modo específico a esta temática y una era accesible gratuitamente en Internet. De sobra, varias eran ya las Universidades que habían instituido cursos en materia del "derecho climático".

A nivel de organización de los gobiernos, existe la tendencia a incluir la política climática en el título del ministerio especializado en el medio ambiente. Éste es el caso de Grecia, dotada desde 2009 hacia enero de 2015 del "Ministerio de Medio Ambiente, Energía y Cambio Climático”. La radiación posterior del cambio climático en el título no significa que el gobierno ha perdido sus competencias relativas a esta cuestión. En todo caso, las mutaciones frecuentes en la denominación de este ministerio y también de otros ha

${ }^{8}$ MANIATIS, A., “The Right to Containing Climate Change (CCC)”, VRONTIS, D., WEBER, Y., TSOUKATOS, E. (Editors), $10^{\text {th }}$ Annual Conference of the EuroMed Academy of Business, Global and national business theories and practice' bridging the past with the future, EuroMed Press, p. 2205, http://euromed2017.com/bop2017.pdf.

${ }^{9}$ DEL GIUDICE, F., Compendio di Diritto Costituzionale, Edizioni Giuridiche Simone 2016, p. 102.

${ }^{10}$ NESPOR, S., “I principi di Oslo: nuove prospettive peri il contenzioso climatico”, Giornale di diritto amministrativo núm. 6, 2015, p. 750. 
causado crítica. Este fenómeno es tan problemático que el nombre de dominio sigue siendo el relativo al cambio climático, es decir “ypeka.gr”.

El Acuerdo de París sobre el cambio climático fue aprobado en la precitada “COP21” el 12 de diciembre de 2015 y entró en vigor muy temprano, en comparación a las otras convenciones internacionales, el 4 de noviembre de 2016. Este hecho tiene una serie de implicaciones, como la entrada en función de su órgano de gobierno, consistente en la Conferencia de las Partes en calidad de reunión en el Acuerdo. Los planes nacionales de acción climática, conocidos formalmente como las contribuciones determinadas a nivel nacional, que las Partes presentaron como aporte a este texto, se transforman en contribuciones determinadas a nivel nacional. En cualquier momento, los países pueden revisar sus planes, únicamente al alza. Los gobiernos tienen también la obligación de tomar medidas para lograr los objetivos establecidos, relativos a la temperatura. Se trata de la limitación del aumento de la temperatura en este siglo a menos de 2 grados centígrados con respecto a los niveles de la era preindustrial y del seguimiento de los esfuerzos para que ese aumento no supere el 1,5 grado centígrado.

La rama del derecho del cambio climático es sobre todo asociada a la Unión europea. Es indicativo el hecho de que ha sido adoptada una mención de este fenómeno en contraposición al derecho comunitario primario antecedente. Según el par. 1 del artículo 191 del Tratado de Funcionamiento de la Unión Europea, la política de la Unión en el ámbito del medio ambiente contribuirá a alcanzar al objetivo de fomento de medidas a escala internacional destinadas a hacer frente a los problemas regionales o mundiales del medio ambiente y en particular a luchar contra el cambio climático. No solamente la Unión ha promovido por excelencia esta rama sino que los Estados Unidos anunciaron en mayo de 2017 su retiro del Acuerdo de París, el cual inflige "severas restricciones de energía” a este país y lo "castiga" mientras "no impone obligaciones significativas a los principales contaminadores del mundo"11. Esta motivación ha sido criticada como errónea dado que la “contribución determinada a nivel nacional” de este Estado ha sido oferta por la administración nacional precedente, es decir no ha sido impuesta por cualquier factor. De sobra, India y China, señaladas en el discurso precitado del Presidente americano, tienen Contribuciones que son distintas de esta Contribución, no porque el Acuerdo imponga un acuerdo prescriptivo para compartir la carga en los países, sino porque estos países tienen circunstancias nacionales diferentes ${ }^{12}$. Debido a razones jurídicas los Estados Unidos se quedaron en este texto hasta noviembre de 2020 y formalmente se retiraron el 4 de ese mes. Sin embargo, este Estado podría volver a unirse al Acuerdo 30 días después de una notificación formal a las Naciones Unidas.

\section{EL PRINCIPIO DE PRECAUCIÓN Y LA JUSTICIA CONSTITUCIONAL EUROPEA}

${ }^{11}$ MANIATIS, A., “The Right...”, p. 2204.

12 RAJAMANI, L., "Reflections on the US withdrawal from the Paris Climate Change Agreement", EJIL, June 5, 2017, https://www.ejiltalk.org/reflections-on-the-us-withdrawal-from-the-paris-climate-changeagreement/. 
El principio de precaución o de cautela ha sido consagrado explícitamente por primera vez por el derecho alemán en la década de 1970, sobre todo en materia del sector de la energía nuclear ${ }^{13}$. A nivel internacional, tiene sus raíces en la Carta Mundial de la Naturaleza, adoptada por la Asamblea General de las Naciones Unidas en 1982. Fue añadido mediante el tratado de la Unión Europea en el artículo 174, hoy el precitado artículo 191 del Tratado de Funcionamiento de la Unión Europea. En la práctica, su ámbito de aplicación es mucho más amplio que el ámbito del medio ambiente y se extiende asimismo a la política de los consumidores, a la legislación europea relativa a los alimentos, a la salud humana, animal y vegetal ${ }^{14}$. Por ejemplo, la legislación alimentaria general, es decir el Reglamento (CE) N. 178/2002, se basa en este principio. Según la Comisión Europea, la cual ha emitido una Comunicación en la materia, de 2.2.2000, la precaución sólo se puede invocar en la hipótesis de un riesgo potencial, y en ningún caso puede justiciar una toma de decisión arbitraria. El recurso a esta herramienta debe guiarse por los siguientes principios específicos:

- Una evaluación científica lo más completa posible y la determinación, en la medida de lo posible, del grado de incertidumbre científica;

- Una determinación del riesgo y de las consecuencias potenciales de la inacción;

- La participación de todas las partes interesadas en el estudio de medidas de precaución, tan pronto como se disponga de los resultados de la evaluación científica o de la determinación del riesgo.

En el ordenamiento francés, el principio precautorio fue adoptado con la ley Barnier, de 2.2.1995. Además, Francia cuenta con una Carta del Medio Ambiente desde 2005, texto con vigencia constitucional. Se trata de un avance importante de la normativa ambiental, la cual se ha promovido del derecho internacional al derecho interno, y mucho más del derecho común, particularmente del derecho administrativo, al derecho constitucional. La Carta afecta entre otros a la legislación, la política gubernamental, la jurisprudencia y el sistema de educación ${ }^{15}$. En 2012, el Consejo de Estado ya había fundado más que 12 sentencias en la Carta, en varios temas ejemplificados por la energía nuclear (como en el caso arquetípico alemán de la precaución) y la protección de los lagos situados en las montañas.

Uno de los principios más relevantes y sobre todo innovadores de este texto es el precautorio. Según el artículo 5, "Cuando la producción de un daño, aunque incierta en el estado de los conocimientos, pueda afectar de manera grave e irreversible al medio ambiente, las autoridades públicas velarán, mediante la aplicación del principio de precaución y en sus ámbitos de competencia, por la implantación de procedimientos de

${ }^{13}$ DE SADELEER, N., Les principes du pollueur-payeur, de prévention et de précaution. Essai sur la genèse et la portée juridique de quelques principes du droit de l'environnement, Bruxelles, Bruylant, 1999, p. 154 ss.

14 EUROPA, Principio de precaución, 2015, http://eur-lex.europa.eu/legalcontent/ES/TXT/?uri=URISERV\%3Al32042.

${ }^{15}$ BOYD, D., "The Constitutional Right to a Healthy Environment”, Environment - Science and Policy

for Sustainable Development, 54, 4, July - August 2012, p. 7, https://www.tandfonline.com/doi/abs/10.1080/00139157.2012.691392. 
evaluación de riesgos y la adopción de medidas provisionales y proporcionadas con el fin de prevenir la producción del daño".

A excepción de Francia, ningún otro Estado de Europa ha hecho una referencia a este principio en su Constitución y, en consecuencia, el caso francés de la Carta del Medio Ambiente parece no haber tenido influencia en los otros países europeos ${ }^{16}$. Sin embargo, la jurisprudencia europea no ha sido indiferente a esta herramienta nueva, aplicada en el marco del derecho internacional y del derecho de la Unión europea.

Por ejemplo, según el par. 1 del artículo 24 de la Constitución griega, enriquecido con las novedades de la durabilidad y del derecho universal ambiental a través de la revisión de 2001, "La protección del medio ambiente natural y cultural constituye obligación del Estado y derecho de cada persona. Para su conservación el Estado tiene obligación de tomar medidas preventivas o represivas particulares en el cuadro del principio de durabilidad". La jurisprudencia del Consejo de Estado, el cual se considera como el substituto del Tribunal Constitucional en el ordenamiento de Grecia, es ambivalente dado que ha procedido a un reconocimiento explícito de la vigencia constitucional del principio precautorio, pero no tiende a hacerlo enseguida, al menos de modo claro. En su sentencia emblemática del derecho ambiental, núm. 613/2002, el tribunal anuló el acto administrativo de aprobación de condiciones ambientales en materia de una fábrica de oro, dentro del sitio arqueológico de Olimpiada (cerca de la ciudad antigua de Aristóteles, en Macedonia), y del lago de las minas de oro. Al ponderar los bienes constitucionales del medio ambiente y del desarrollo (económico), favoreció el primero, haciendo uso de términos no meramente de la Constitución sino también del derecho comunitario, en su motivación. Paralelamente, formuló directamente el respecto al principio de prevención y precaución del medio ambiente. Se trata de una sentencia muy relevante, la cual reconoció el doble principio preventivo, es decir de acción preventiva y de precaución, en base de la disposición precitada del texto constitucional ${ }^{17}$.

Sin embargo, el mismo tribunal ha evitado reiterar este reconocimiento, por ejemplo, en su sentencia núm. 101/2018, en la cual hace mención de la precaución en el Tratado de Funcionamiento de la Unión europea y confirma la existencia del principio de obligación de restauración de los ecosistemas importantes sustancialmente dañados, como un río. Cabe señalar que ambas herramientas, la restaurativa y la precautoria, resultan de la misma disposición de la Constitución y que la primera debe aplicarse de modo previo a la segunda, en el marco del control de una nueva carga eventual del ecosistema ya dañado. Restauración, precaución y prevención constituyen así un sistema unitario de operación circular. Como el derecho administrativo general en una fase pasada, el derecho ambiental sigue siendo, en un grado elevado, un derecho de proveniencia jurisprudencial, sobre todo de la obra del Consejo de Estado.

${ }^{16}$ CHIU, V., "Les cours constitutionnelles européennes et le principe de précaution”, rfda, novembredécembre 2017, p. 1055.

17 MANIATIS, A., Trabajos de protección de monumentos y la guerra contra el saqueo de antigüedades, Ediciones Ant. N. Sákkoula Atenas - Komotiní, 2010, págs. $75-76$ (en griego). 
Por lo que se refiere a los países europeos dotados de jurisdicción especializada para ejercer el control de constitucionalidad de las disposiciones legislativas, ella se enfrentó al principio precautorio principalmente en materia ambiental ${ }^{18}$. El principio es raramente aplicado, dada la dificultad de captar su contenido. Si los jueces constitucionales en su mayoría son muy reacios a aplicarlo, algunos han podido incorporarlo en el marco del control de constitucionalidad. En los casos que el juez decide reconocer una vigencia constitucional a la precaución, la acción del legislador es limitada por ella ${ }^{19}$. Este principio constitucional ofrece al legislador la posibilidad de apreciar ampliamente la existencia de varios riesgos, para autorizar o prohibir ciertas actividades.

\section{LA CUESTIÓN DE PROHIBICIÓN DEL FRACKING Y LA JUSTICIA CONSTITUCIONAL EUROPEA}

Francia, invocando la precaución, se convirtió en el primer Estado por el mundo entero que prohibió el recurso al método de craqueo hidráulico. Este término significa un proceso que consiste en extracción de combustibles fósiles (sea gas sea petróleo) atrapados en rocas solidas a través de la canalización, bajo presión, de una mezcla de agua, arena y aditivos químicos para causar pequeñas grietas en las rocas y así liberar el depósito. El Consejo Constitucional declaró, el 11 de octubre de 2013, que la ley prohibitiva, votada el 13 de julio de 2011, era conforme a la Constitución. Por el contrario, la empresa americana interesada había expresado la posición que la terminación de la vigencia de sus autorizaciones de exploración y de explotación constituía una aplicación "demasiado estricta” del principio de la precaución.

Esta jurisprudencia resultó del procedimiento de "Cuestiones Prejudiciales de Constitucionalidad", el cual funciona desde 2010. La poca utilización de este mecanismo en materia del medio ambiente no podría explicarse solamente en base de razones procedurales. En el contencioso civil, cuando los grandes principios del derecho ambiental deben aplicarse, la ley Barnier, incluida en los artículos 110-1 y 110-2 del Código del Medio Ambiente, es muy frecuentemente tomada en cuenta dado que ella siembra a veces más precisa, sobre todo en materia de la prevención o de la precaución ${ }^{20}$. En todo caso, la adopción del Código ha fortalecido el carácter autónomo de la rama del derecho ambiental.

Además, la jurisdicción constitucional de España y de Lituania se niega a aplicar el principio precautorio y lo confunde con el principio de prevención, destinado a proteger contra riesgos de certidumbre ${ }^{21}$. El tribunal constitucional español en la sentencia núm. 106/2014 y su homólogo lituano en la sentencia de 16 de diciembre de 2015 en materia del uso del craqueo hidráulico para la exploración y extracción del gas de esquisto no reconocieron el derecho ecológico específico a la protección contra el fracking. De acuerdo con los solicitantes, el uso de este método incluye los riesgos probados en detrimento del medio ambiente y de la salud e igualmente otros riesgos potenciales, sobre todo riesgos de contaminación de las aguas subterráneas y sísmico. Resulta que la cuestión de su

\footnotetext{
${ }^{18}$ CHIU, V., “Les cours...”, cit., p. 1055.

${ }^{19}$ CHIU, V., "Les cours...”, cit., p. 1060.

${ }^{20} \mathrm{CH}$. HUGLO, “La QPC...”, cit.

${ }^{21}$ CHIU, V., “Les cours...”, cit., pp. 1059-1060.
} 
prohibición ha sido indirectamente asociada a la garantía del agua, la cual constituye un derecho fundamental de tercera generación ${ }^{22}$. Ambos tribunales no hicieron referencia a la existencia de una certidumbre o incertidumbre científica relativa a este uso. Sus dos sentencias, altamente cuestionables, parecen estar justificadas por un contexto de crisis económica y energética.

\section{EL PRINCIPIO DE ENDOGENEIDAD AMBIENTAL DEL TURISMO}

En materia de España y particularmente de Cantabria, según la doctrina, no es fácilmente comprensible cómo se puede impedir que una Comunidad Autónoma apueste decididamente por el turismo sostenible como ámbito económico y consecuentemente prohíba en su territorio prácticas ambientalmente inciertas y dudosas como el fracking, en base a un entendimiento economicista del concepto «energía» ${ }^{23}$.

El turismo sigue siendo un derecho marginal, por lo que se refiere a su positivación constitucional, mucho más a su correlación con el medio ambiente en las Constituciones. Sin embargo, la Constitución actual de la República Bolivariana de Venezuela, adoptada en su forma inicial en 1999, incluye la garantía del turismo, mucho más con un modo enfático. Más precisamente, el artículo 156 numeral 23 hace mención que entre las competencias del Poder Público Nacional se encuentra estipulado el fomento a las políticas nacionales y la legislación en materia de turismo. Si alguien podría observar que este artículo se refiere a muchos temas heteróclitos, la importancia del turismo se destaca a través de la existencia del artículo 310, totalmente dedicado a este tema. Según este artículo, "El turismo es una actividad económica de interés nacional, prioritaria para el país en su estrategia de diversificación y desarrollo sustentable. Dentro de las fundamentaciones del régimen socioeconómico previsto en esta Constitución, el Estado dictará las medidas que garanticen su desarrollo. El Estado velará por la creación y fortalecimiento del sector turístico nacional”.

La doctrina ha comentado que al hablarse que es una actividad económica de interés nacional, la propia normativa constitucional se enfocó en dos conceptos, primeramente en lo sostenible y en segundo lugar que sea endógeno, ya que la actividad turística debe conservar el medio ambiente, por ello es necesario contar con innovaciones en la prestación del servicio turístico, con la finalidad de prevenir los agravios ocasionados por estas actividades; éste es el avance que esta disposición trajo en la normativa venezolana, tratar de enfocar a la actividad turística en gestionar desde una perspectiva más humana los recursos, su producción y servicios ${ }^{24}$.

Es también notable que el principio genérico de desarrollo sostenible es aplicable en materia de turismo, a nivel mundial. Éste el caso sobre todo del Código Ético Mundial

${ }^{22}$ MANIATIS, A., El Derecho al Agua y la Contratación Pública. Estudios de Derecho Público, Editorial Académica Española, 2018, p. 3.

${ }^{23}$ GALERA RODRIGO, S., "En el día del medio ambiente: España, ¿Hasta cuándo el último de la clase en políticas ambientales europeas”, Actualidad Jurídica Ambiental, n. 80, 5 de junio de 2018, pp. 3-4.

${ }^{24}$ TORREALBA DUGARTE, J. D., "Fundamentos Para el Desarrollo del Derecho Turístico En Venezuela Como Rama Jurídica Autónoma”, Digiciencia UDEFA Vol. 2, Octubre 2013, p. 13, http://www.udefa.edu.ve/digiciencia/vol2/digiciencia_vol2.pdf. 
para el turismo, el cual enfatiza en la dimensión ambiental de esta actividad. No obstante, el hecho de que el turismo tiene una relación estrecha con el medio ambiente no se limita a la cuestión de respecto de los bienes ambientales por los turistas y las empresas de la industria turística. En realidad, el turismo constituye un fenómeno endógeno del medio ambiente, en un sentido más avanzado. Se trata de una actividad que tiene lugar en el marco de la naturaleza y de los bienes culturales y está particularmente vinculado al patrimonio cultural $^{25}$.

La actividad turística es comparable con aquella de los deportes, no solamente porque ambos fenómenos consisten en un uso agradable del tiempo libre a través del movimiento del cuerpo humano. Implica desplazamiento de la persona involucrada para entrar en contacto con la naturaleza y también los otros recursos turísticos y tiene una relativa independencia frente a la producción de productos y prestación de servicios ${ }^{26}$. Se trata de una actividad privada de consumidores, es decir por razones no productivas, en directa comunicación con el medio ambiente en conjunto.

Además, el hombre tiene una relación intrínseca y directa con la naturaleza y los otros recursos turísticos, independientemente de sus cualidades personales como la ciudadanía y la residencia. Según el artículo 4 par. 1 del precitado Código, “Los recursos turísticos pertenecen al patrimonio común de la humanidad. Las comunidades en cuyo territorio se encuentran tienen con respecto a ellos derechos y obligaciones particulares". Estas disposiciones destacan el carácter universal del derecho fundamental al turismo y fomentan el turismo internacional sin despreciar la relación de cada comunidad local con su entorno.

Es indicativo de la particularidad del fenómeno del turismo que muchas veces se ejerce particularmente para conocer a la gente de la sociedad del destino en lugar de los bienes culturales y quizá también de los aspectos de la naturaleza ${ }^{27}$. Resulta el pluralismo del contenido del medio ambiente, el cual incluye sobre todo los seres humanos. De sobra, se destaca el concepto de la amistad entre los distintos pueblos y más ampliamente de la hospitalidad.

El fenómeno del turismo es relacionado con el sector terciario de la economía y no consiste en un ejemplo del sector secundario, pese a la tendencia general a hablar de una industria. En todo caso, la "industria turística" se considera más respetuosa con el medio ambiente que la industria auténtica ${ }^{28}$. Éste es el caso de una actividad turística que tiene lugar sobre todo en grandes ciudades, que presumiblemente están amenazadas por diversas formas de degradación ambiental. Se trata de los recorridos guiados, ejemplificados por los tours gratis (o free tours), que constituyen prácticas de actividad turística particularmente amigables con el medio ambiente por varias razones.

${ }^{25}$ MANIATIS, A., CORTÉS-RAMÍREZ E. E., El Derecho a la Información Turística. La Inversión del Papel del Guía, Editorial Académica Española, 2020, p. 163.

${ }^{26}$ MANIATIS, A., CORTÉS-RAMÍREZ E. E., El Derecho..., cit., p. 163.

${ }^{27}$ MANIATIS, A., CORTÉS-RAMÍREZ E. E., El Derecho..., cit., p. 163.

${ }^{28}$ MANIATIS, A., CORTÉS-RAMÍREZ E. E., El Derecho..., cit., p. 164. 
Dado que la endogeneidad del turismo es una realidad social, se ha propuesto que ella se consagre legalmente, como principio genérico del derecho turístico ${ }^{29}$. Según este acercamiento, podría compararse y contrastarse con el principio de integración de las políticas ambientales, en sectores de producción económica, como el comercio y la industria, en el marco del derecho de la Unión europea. Por un lado, actividades las cuales sobre todo o plenamente carecen de carácter productivo, como las recreativas, se realizan en contacto directo con la naturaleza y los bienes culturales de manera que están intrínsecamente integradas al medio ambiente. Resulta que el turismo, como la actividad recreacional del deporte, debería estar sujeta a una nueva garantía genérica del derecho ambiental, consistente en la endogeneidad del uso no productivo de los bienes del medio ambiente. La emergencia de esta novedad no es sencillamente un hecho producido por la investigación, sino que podría tener ciertas consecuencias en la práctica. Es obvio que este principio implicaría una prioridad, conceptual y práctica, de ese tipo de uso frente a la producción, como es el caso del sector secundario. Por otro, los tres sectores de la economía deben estar permanentemente vinculados a la política de protección del entorno de los seres humanos, en particular al principio central de durabilidad. El concepto más avanzado que sugiere una cierta externalidad frente al medio ambiente, el cual se considera como exclusivamente material, consiste en la aproximación a las energías como una entidad autónoma. Esta distinción conceptual no es correcta dado que el entorno es único y polivalente, incluyendo los seres humanos (sobre todo, como ya señalado), las demás entidades materiales y también varias formas de energía, particularmente la energía solar. En conclusión, la endogeneidad ecológica del turismo no solamente rige el turismo, sino que también le da prioridad sobre otros sectores o subsectores a causa de su carácter amigable para la naturaleza y su inmediatez con respecto a las políticas ambientales.

\section{EL DERECHO AMBIENTAL EN LA VANGUARDIA DEL DERECHO CONSTITUCIONAL}

El derecho ambiental es dotado de varios principios típicos de su fisonomía, como la precaución y la durabilidad. No es casualidad que ambos principios hayan recientemente adquirido vigencia constitucional, al menos mediante la contribución de la jurisprudencia, en los ordenamientos de Grecia y Francia. Por lo que se refiere a la durabilidad, ella constituye una garantía general, la cual es directamente aplicable no solamente en el medio ambiente sino también en el sector turístico.

De sobra, sería relevante destacar la endogeneidad ambiental de varias actividades relacionadas con la recreación (turismo, deporte...), la cual consiste en un concepto de proveniencia doctrinal y podría consagrarse de manera explícita en el derecho positivo. Particularmente por lo que se refiere a los desplazamientos turísticos, la precitada relación posesiva de cada persona con el medio ambiente facilita la introducción del principio de endogeneidad ambiental del turismo. Por tanto, el turismo merecería estar a la vanguardia de la lucha para proteger el medio ambiente.

Otra garantía central del derecho ecológico consiste en el principio genérico de prevención, el cual incluye los principios especiales de precaución, de prevención (en

${ }^{29}$ MANIATIS, A., CORTÉS-RAMÍREZ E. E., El Derecho..., cit., p. 164. 
sentido estricto) o acción preventiva y de restauración de ecosistemas importantes sustancialmente dañados.

Francia ha promocionado el derecho ambiental y el derecho constitucional, con énfasis en la participación social a la toma de decisiones. Este pluralismo es indicativo de la nueva constelación de derechos relativos al medio ambiente. Por el contrario, el sistema jurídico y administrativo de Grecia es esencialmente ajeno a una participación pluralista en la toma de decisiones ${ }^{30}$. Todos los períodos de la historia de la gobernanza de posguerra, en el sector de las aguas, son indicativos de ese esoterismo de los órganos del poder público. Esta historia está caracterizada por la implementación incompleta del marco institucional de la política hídrica ${ }^{31}$.

Además, el avance en la constelación de derechos relativos al medio ambiente está ejemplificado por el caso del principio precautorio (derecho ambiental y también derecho constitucional material) y por el procedimiento de Cuestiones Prejudiciales de Constitucionalidad (derecho constitucional).

El derecho ecológico constituye la vanguardia del derecho constitucional, particularmente en materia de los derechos fundamentales, y el derecho al medio ambiente es ejemplo típico de la teoría de los llamados "nuevos derechos", es decir facultades no previstas, al menos explícitamente, en las Constituciones ${ }^{32}$. Se trata de la componente central de los derechos de solidaridad en tal punto que esa onda de tercera generación, de la que forma parte el derecho al desarrollo sostenible, se considera sobre todo como “ambiental" 33 .

El derecho ambiental no podría considerarse irrelevante para los derechos fundamentales de cuarta generación, como es sobre todo la mitigación del cambio climático $^{34}$. La normativa climática emergió en forma de subrama de este derecho, pero hoy día podría considerarse como rama autónoma, desde la conclusión del Acuerdo de París. Este desarrollo es indicativo del carácter dramático del problema del cambio climático y también de la dinámica de la normativa ecológica. De sobra, es notable que los derechos de la tercera generación y aquellos de la cuarta tienen características comunes, como la solidaridad, la universalidad y el carácter colectivo.

En el marco del derecho constitucional europeo y global sería recomendable proceder en las Constituciones a la prohibición del uso del fracking y al reconocimiento expreso de los derechos fundamentales universales al Sol; se trataría sobre todo del derecho a la mitigación del cambio climático, agrupado con el derecho al Sol (en sentido estricto), cuya subcategoría es el derecho a la energía solar, también llamado “derecho solar”.

30 MANIATIS, A., NTAVRI, A., TSIPOURIARI, V., “La política ambiental de Grecia”, Anu Fac Der UDC, 23, 2019, p. 197.

${ }^{31}$ KRITSOTAKIS, M., “Gobernanza de agua y sector agrícola”, Epí Gis, núm. 10 Otoño 2017, p. 23 (en griego), https://www.piraeusbank.gr/ /media/Gr/Agrotes/Files/Epi-Gis/EPI-GHS-10.pdf.

32 DEL GIUDICE, F., Compendio..., cit., p. 109.

33 MANGION, R., "Four generations of human rights", Timesofmalta.com, June 14, 2013, http://www.timesofmalta.com/articles/view/20130614/opinion/Four-generations-of-human-rights.473785.

${ }^{34}$ MANIATIS, A., “4G Rights and Criminality”, Forensic Sci Add Res, 3(1), FSAR.000556.2018, p. 1. 


\section{BIBLIOGRAFÍA}

BOYD, D., “The Constitutional Right to a Healthy Environment”, Environment - Science and Policy for Sustainable Development, 54, 4, July - August 2012, pp. 3-14, https://www.tandfonline.com/doi/abs/10.1080/00139157.2012.691392.

CHIU, V., "Les cours constitutionnelles européennes et le principe de précaution", rfda, novembre-décembre 2017.

EUROPA, Principio de precaución, 2015, http://eur-lex.europa.eu/legalcontent/ES/TXT/?uri=URISERV\%3Al32042.

GALERA RODRIGO, S., "En el día del medio ambiente: España, ¿Hasta cuándo el último de la clase en políticas ambientales europeas”, Actualidad Jurídica Ambiental, n. 80, 5 de junio de 2018.

DEL GIUDICE, F., Compendio di Diritto Costituzionale, Edizioni Giuridiche Simone 2016.

DE LANVERSIN, J., 'Contribution du juge au développement du droit de l'environnement', en Mélanges M. Waline - Le juge et le droit public, T. II 1974.

DE SADELEER, N., Les principes du pollueur-payeur, de prévention et de précaution. Essai sur la genèse et la portée juridique de quelques principes du droit de l'environnement, Bruxelles, Bruylant, 1999.

FEIJÓO REY, M. J., PIMENTEL SILES, M. (Editores), El interés general y la excelencia en los servicios públicos. La mejor gestión de los servicios municipales en favor de los ciudadanos y no de las ideologías, Almuzara, 2017.

HUDSON, B., "Structural Environmental Constitutionalism”, Journal Articles. 2015, https://digitalcommons.law.lsu.edu/faculty_scholarship/170.

HUGLO, CH., “La QPC: quelle utilisation en droit de l'environnement?”, Nouveaux Cahiers du Conseil Constitutionnel, n. 43 (le Conseil constitutionnel et l'environnement) avril 2014, https://www.conseil-constitutionnel.fr/nouveaux-cahiers-du-conseilconstitutionnel/la-qpc-quelle-utilisation-en-droit-de-l-environnement.

KRITSOTAKIS, M., “Gobernanza de agua y sector agrícola”, Epí Gis, núm. 10, Otoño 2017 (en griego), https://www.piraeusbank.gr/ /media/Gr/Agrotes/Files/Epi-Gis/EPI-GHS10.pdf. 
MANGION, R., "Four generations of human rights", Timesofmalta.com, June 14, 2013, http://www.timesofmalta.com/articles/view/20130614/opinion/Four-generations-of-humanrights.473785.

MANIATIS, A., "4G Rights and Criminality", Forensic Sci Add Res, 3(1), FSAR.000556.2018.

MANIATIS, A., El Derecho al Agua y la Contratación Pública. Estudios de Derecho Público, Editorial Académica Española, 2018.

MANIATIS, A., CORTÉS-RAMÍREZ E. E., El Derecho a la Información Turística. La Inversión del Papel del Guía, Editorial Académica Española, 2020.

MANIATIS, A., NTAVRI, A. TSIPOURIARI, V., "La política ambiental de Grecia”, Anu Fac Der UDC, 23, 2019.

MANIATIS, A., "PPP and the constitutional right to the environment", Constitutionalism.gr, 20.11.2015, $\quad$ https://www.constitutionalism.gr/ppp-and-theconstitutional-right-to-the-environment/?hilite=\%27Antonios\%27\%2C\%27Maniatis\%27.

MANIATIS, A., "The Right to Containing Climate Change (CCC)", VRONTIS, D., WEBER, Y., TSOUKATOS, E. (Editors), $10^{\text {th }}$ Annual Conference of the EuroMed Academy of Business, Global and national business theories and practice' bridging the past with the future, EuroMed Press, http://euromed2017.com/bop2017.pdf.

MANIATIS, A., Trabajos de protección de monumentos y la guerra contra el saqueo de antigüedades, Ediciones Ant. N. Sákkoula Atenas - Komotiní, 2010 (en griego).

NESPOR, S., "I principi di Oslo: nuove prospettive per il contenzioso climatico", Giornale di diritto amministrativo núm. 6, 2015.

RAJAMANI, L., "Reflections on the US withdrawal from the Paris Climate Change Agreement”, EJIL, June 5, 2017, https://www.ejiltalk.org/reflections-on-the-us-withdrawalfrom-the-paris-climate-change-agreement/.

ROMANO, A., "Los principios constitucionales de las Administraciones Públicas”, ReDCE núm. 23, 2015 (trad. PÉREZ MIRAS, A.).

SIOUTI, G., La consagración constitucional de la protección del medio ambiente, Ediciones Ant. N. Sákkoula Atenas - Komotiní 1985 (en griego).

TORREALBA DUGARTE, J. D., "Fundamentos Para el Desarrollo del Derecho Turístico En Venezuela Como Rama Jurídica Autónoma”, Digiciencia UDEFA Vol. 2, Octubre 2013, http://www.udefa.edu.ve/digiciencia/vol2/digiciencia_vol2.pdf. 